\title{
Guía de práctica clínica para la prevención y manejo del parto pretérmino en el Seguro Social del Perú (EsSalud), 2018
}

\author{
Peruvian Social Security (EsSalud) 2018 Clinical \\ Practice Guidelines for prevention and management \\ of preterm births
}

Correspondencia Raúl Timaná-Ruiz Jirón Domingo Cueto 109, Jesús María, Lima, Perú Teléfono: (051) 2656000 anexo: 1953

rtimanar@gmail.com

\section{Recibido: 21/01/2019}

Arbitrado por pares

Aprobado: 28/02/2019

Citar como: Yanque-Robles O, ZafraTanaka JH, Taype-Rondan A, ArroyoCampuzano JE, Rosales-Cerrillo CH, Mucha J, et al. Guía de práctica clínica para la prevención y manejo del parto pretérmino en el Seguro Social del Perú (EsSalud), 2018. Acta Med Peru. 2019;36(1):46-56
Omar Yanque-Robles', Jessica H. Zafra-Tanaka², Alvaro Taype-Rondan², Jorge E. Arroyo-Campuzano ${ }^{3}$, César H. Rosales-Cerrillo3, Jorge Mucha ${ }^{4}$, Raúl C. AlegriaGuerrero ${ }^{3}$, Martina Zegarra ${ }^{1}$, Marisol O. Ramírez-Linares ${ }^{1}$, Ana C. Lino-Salazar ${ }^{3}$, Pablo Moreno ${ }^{6}$, Daniel S. Ramos-Espinoza ${ }^{5}$, Manuel A. Caballero-Noriega ${ }^{5}$, Gastón C. Chumbipuma-Salsavilca ${ }^{7}$, Larissa Otero ${ }^{8}$, Alberto Guerra-García ${ }^{8}$, Naysha Becerra-Chauca² ${ }^{2}$ Stefany Salvador-Salvador ${ }^{2}$, Raúl Timaná-Ruiz²

1 Hospital Nacional Edgardo Rebagliati Martins, EsSalud. Lima, Perú.

2 Instituto de Evaluación de Tecnologías en Salud e Investigación, EsSalud. Lima, Perú.

3 Hospital Nacional Alberto Sabogal Sologuren, EsSalud. Lima, Perú.

4 Hospital Nacional Guillermo Almenara Irigoyen, EsSalud. Lima, Perú.

5 Hospital III Suárez Angamos, EsSalud. Lima, Perú.

6 Hospital Guillermo Kaelin de la Fuente. Lima, Perú

7 Hospital de Emergencias III Grau, EsSalud. Lima, Perú

8 Universidad Peruana Cayetano Heredia. Lima, Perú

\section{RESUMEN}

El presente artículo resume la guía de práctica clínica (GPC) para la prevención y manejo del parto pretérmino en el Seguro Social del Perú (EsSalud). Para el desarrollo de esta GPC, se conformó un grupo elaborador de la guía (GEG) que incluyó especialistas clínicos y metodólogos, el cual formuló 11 preguntas clínicas. Para responder cada pregunta se realizó búsquedas sistemáticas en Pubmed y en repositorios de GPC, y se seleccionó la evidencia pertinente. La certeza de la evidencia fue evaluada usando la metodología Grading of Recommendations Assessment, Development, and Evaluation (GRADE). En reuniones periódicas, el GEG usó la metodología GRADE para revisar la evidencia y emitir las recomendaciones. Se emitieron 20 recomendaciones (13 fuertes y 7 condicionales), 24 puntos de buena práctica clínica, una recomendación de implementación y un flujograma.

Palabras clave: Trabajo de parto prematuro; Guía de práctica clínica; Medicina basada en evidencias (fuente: DeCS BIREME). 


\begin{abstract}
This paper features a summary of the Peruvian Social Security (EsSalud) Clinical Practice Guidelines (CPG) for prevention and management of preterm birth. A specialized group was formed for writing this CPG, which included clinical and methodology specialists, who formulated 11 clinical questions. Systematic searches in PubMed and CPG repositories were performed aiming to answer the questions, and relevant evidence was selected. The certainty of the evidence was assessed using the Grading of Recommendations Assessment, Development, and Evaluation (GRADE) methodology. In regular work meetings, the specialized group used the GRADE approach for reviewing the evidence and for developing recommendations. At the end, this CPG formulated twenty recommendations (13 strong and 7 conditional), 24 good clinical practice points, one recommendation for implementation, and a flowchart.
\end{abstract}

Keywords: Obstetric labor, premature; Practice guideline; Evidence-based medicine (source: MeSH NLM).

\section{INTRODUCCIÓN}

Se define como parto pretérmino (PPT) a aquel que sucede antes de las 37 semanas de gestación. Se subdivide en pretérmino extremo (antes de las 28 semanas), muy pretérmino (entre 28 y antes de 32 semanas) y pretérmino tardío (entre las 32 y antes de las 37 semanas) ${ }^{[1]}$.

Se estima que en el año 2010 el 11,1\% de los partos a nivel mundial fueron pretérmino, lo que se tradujo en 14,9 millones (intervalo de confianza al 95\% [IC 95\%]: 12,3 - 18,1 millones), la mayoría de ellos $-12,5$ millones- ocurrió después de las 32 semanas ${ }^{[1]}$. En Latinoamérica, para el año 2010, la tasa de PPT llegó al 8,4\% del total de partos, lo que equivalió a 853000 (IC 95\%: $696000-1164000$ ) partos pretérmino ${ }^{[1]}$. En el Perú, para julio del 2017 , el $25,43 \%$ de las defunciones neonatales fueron por causas relacionadas al PPT ${ }^{[2]}$.

Estas cifras son preocupantes pues los recién nacidos pretérmino (RN PT) tienen un mayor riesgo de morir durante el periodo neonatal, desarrollar infecciones, o padecer parálisis cerebral, déficit sensorial, discapacidades de aprendizaje y enfermedades respiratorias; comparados con los nacidos a término; además, la incidencia de estas complicaciones se incrementa cuanto menor sea la edad gestacional al nacimiento. Asimismo, cabe resaltar los elevados costos económicos que conlleva en términos de cuidados intensivos neonatales, atención médica continua y necesidades educativas a largo plazo ${ }^{[1,3]}$.

Por lo mencionado, el PPT es un problema clínico relevante, cuya morbimortalidad neonatal se puede reducir con adecuadas medidas de prevención y manejo ${ }^{[4]}$. Por esta razón, el Seguro Social de Salud del Perú (EsSalud) estableció recomendaciones basadas en evidencias para guiar los procesos y procedimientos de prevención y manejo del PPT.

\section{METODOLOGÍA}

El procedimiento seguido para la elaboración de la presente guía de práctica clínica (GPC) se encuentra detallado en la página web del Instituto de Evaluación de Tecnologías en Salud e Investigación (IETSI) de EsSalud (ver: http://www.essalud. gob.pe/ietsi/guias pract clini.html). De manera resumida, se describen los pasos seguidos:

\section{Conformación del grupo elaborador de la guía (GEG)}

La Dirección de Guías de Práctica Clínica, Farmacovigilancia y Tecnovigilancia del IETSI conformó el GEG, el cual incluyó a expertos en la metodología de elaboración de GPC y médicos especialistas en el tema (ginecoobstetras y pediatras neonatólogos).

\section{Formulación de preguntas}

En concordancia con los objetivos y alcances de la GPC, se formularon 11 preguntas clínicas (Tabla 1), que incluyeron una o más preguntas PICO (del inglés: Population, Intervention, Comparator, Outcome). El GEG definió los desenlaces críticos e importantes para cada pregunta PICO (Anexo 1, disponible en la versión virtual).

\section{Búsqueda y selección de la evidencia}

Se realizó la búsqueda sistemática y selección de la evidencia para cada pregunta PICO. Para ello, durante el 2017 se buscaron revisiones sistemáticas (RS) publicadas como artículos científicos (en Pubmed) o realizadas como parte de una GPC (en bases de datos y repositorios de GPC). Con respecto a las GPC, se encontraron tres que realizaron RS y tuvieron no más de cinco años de antigüedad: Preterm labour and birth del National Institute for Health and Care Excellence (NICE) 2015 ${ }^{[5]}$, Recommendations on interventions to improve preterm birth outcomes de la Organización Mundial de la Salud (OMS) 2015 ${ }^{[6]}$, y Prevention of preterm birth in women at risk: selected topics del Belgian Health Care Knowledge Centre (KCE) $2014{ }^{[7]}$. Posteriormente, se seleccionaron las RS (ya sean publicadas como artículos científicos o como parte de una GPC), las cuales fueron actualizadas cuando fue considerado necesario. Finalmente, se evaluó el riesgo de sesgo de los estudios incluidos que fueron encontrados en la actualización. Cuando no se encontró ninguna RS para cierta pregunta, se realizó una búsqueda de novo de estudios primarios (Anexo 2, disponible en la versión virtual). 


\section{Evaluación de la certeza de la evidencia}

Para evaluar la certeza de la evidencia, se siguió la metodología de Grading of Recommendations Assessment, Development, and Evaluation (GRADE) ${ }^{[8]}$. Se construyeron nuevas tablas de perfil de evidencia GRADE en aquellos desenlaces que no tuvieran alguna en investigaciones previas (publicadas en una RS o GPC), o cuando las existentes presentaban inconsistencias o no se adecuaban a nuestro contexto (Anexo 3, disponible en la versión virtual).

\section{Formulación de las recomendaciones}

Se realizaron reuniones periódicas en las cuales el GEG presentó la evidencia a médicos expertos clínicos con el fin de que, con base en estas, se emitan recomendaciones, ya sea de forma unánime o por mayoría simple. Este proceso obedeció a los dispuesto por la metodología GRADE ${ }^{[8]}$, que valora los beneficios y riesgos de las opciones, la certeza de la evidencia, los valores y preferencias de los pacientes y el uso de recursos. Además, se evaluó la aceptabilidad y factibilidad de las recomendaciones, con miras a su implementación. Cada recomendación fue emitida con una dirección (a favor o en contra) y fuerza (fuerte o condicional) específica. De considerarse pertinente, se formularon puntos de buena práctica clínica (BPC) por consenso, o enunciados sobre la implementación de nuevas tecnologías en EsSalud -conocidos como notas de implementación (NI)-.

\section{Revisión}

La presente GPC fue revisada en reuniones con médicos especialistas representantes de otras instituciones, pacientes y responsables de la toma de decisiones. Asimismo, su versión extensa fue enviada por vía electrónica a tres expertos (un ginecoobstetra, un neonatólogo y un revisor externo) para su revisión. El GEG tuvo en cuenta los resultados de estas revisiones para modificar las recomendaciones finales.

\section{Aprobación de la GPC}

La presente GPC fue aprobada para su uso en EsSalud, con Resolución Nº 138-IETSI-ESSALUD-2018.

\section{Actualización de la GPC}

Esta GPC tiene una validez de tres años. Al acercarse al fin de este período, se procederá a realizar una RS de la literatura para su actualización, luego de la cual se decidirá si es pertinente ampliar el periodo de validez, actualizar la GPC o realizar una nueva versión.

\section{Evidencia y recomendaciones}

La presente GPC desarrolló 11 preguntas clínicas divididas en tres temas: prevención secundaria, diagnóstico y manejo del PPT. Con base en ellas se formularon 20 recomendaciones (13 fuertes y siete condicionales), 24 puntos de BPC, una NI (Tabla 1) y un flujograma (Figura 1). A continuación, se resume el razonamiento seguido para llegar a cada recomendación, BPC y NI.
Pregunta 1. En gestantes con riesgo de PPT, ¿se debería usar progesterona o cerclaje como prevención secundaria?

EI GEG estableció dos grupos de riesgo: mujeres con antecedente de haber tenido PPT o tener cérvix corto. Para estos casos, se evaluó dos terapias profilácticas: uso de progesterona y cerclaje.

Con respecto a la terapia profiláctica con progesterona, se consideró la evidencia presente en la GPC KCE $2014{ }^{[7]} \mathrm{y}$ la RS de Romero $2012^{[9]}$. En gestantes con cérvix corto, la progesterona disminuyó el riesgo de PPT y de síndrome de distrés respiratorio (SDR), y no se encontró efecto sobre muerte neonatal o perinatal ni enterocolitis necrotizante, por lo que se emitió una recomendación a favor de su uso. Debido a que los desenlaces de interés solo fueron evaluados por un estudio, esta recomendación fue condicional.

Por otro lado, en gestantes con antecedente de PPT espontáneo, la progesterona disminuyó el riesgo de PPT y no se encontró efecto en desenlaces perinatales adversos, por lo que también se emitió una recomendación a favor de su uso. Debido a que los beneficios potenciales eran importantes y fueron replicados en diferentes estudios, esta recomendación fue fuerte.

Con respecto la terapia profiláctica con cerclaje, se consideró la evidencia de la GPC NICE $2015^{[5]}$ y la GPC KCE $2014{ }^{[7]}$. En gestantes con cérvix corto, el cerclaje disminuyó el riesgo de PPT pero aumentó el riesgo de pirexia; en tanto que en las gestantes con antecedente de PPT, el cerclaje no tuvo efecto sobre el riesgo de PPT ni de desenlaces perinatales adversos. El GEG consideró que, existiendo como alternativa la progesterona vaginal -que es un procedimiento más sencillo, factible y con menos efectos adversos-, se debería priorizar dicho manejo, por lo que decidió no emitir una recomendación sobre el cerclaje.

Por último, el panel decidió emitir dos puntos de BPC, uno con respecto a brindar información y otro en relación al momento y procedimiento usado para la identificación del cérvix corto.

Pregunta 2. En gestantes en las que se sospeche de ruptura prematura de membranas (RPM) pretérmino, ¿qué prueba se debería usar para realizar su diagnóstico?

El GEG decidió evaluar tres pruebas para el diagnóstico del RPM pretérmino en pacientes sin signos clínicos: alfa microglobulina -1 (PAMG-1, por sus siglas en inglés), la prueba de proteína similar a insulina unida al factor de crecimiento (IGFBP-1, por sus siglas en inglés) y la alfa-fetoproteína (AFP).

La RS de Palacio $2014{ }^{[10]}$ encontró que, para el diagnóstico de RPM, el PAMG-1 tuvo una sensibilidad de $96,8 \%$ y una especificidad de $98,3 \%$, mientras que IGFBP-1 tuvo una sensibilidad de $92,1 \%$ y una especificidad de $90,5 \%$. El estudio de Eleje $2017^{[11]}$ encontró que, para el diagnóstico de RPM, la AFP presentaba una sensibilidad de $95,8 \%$ y especificidad de 95,5\%, mientras que la combinación de AFP + IGFBP-1 tuvo un sensibilidad de $97,9 \%$ y una especificidad de $97,6 \%$. 
Tabla 1. Lista de preguntas clínicas, recomendaciones, puntos de buena práctica clínica y notas de implementación.

\section{Enunciado}

\section{Pregunta 1. En gestantes con riesgo de parto pretérmino (PPT), ¿se debería usar progesterona o cerclaje como prevención secundaria?}

En gestantes asintomáticas con antecedente de PPT espontáneo, recomendamos indicar progesterona vaginal micronizada ( $200 \mathrm{mg}$, una vez al día [durante la noche] desde el inicio del segundo trimestre en adelante o por lo menos hasta la semana 34).

En gestantes asintomáticas con cérvix corto identificado en ecografía trasvaginal sugerimos progesterona vaginal micronizada (200 mg, una vez al día [durante la noche] desde el inicio del segundo trimestre en adelante o por lo menos hasta la semana 34).

Brindar información y apoyo a gestantes con riesgo de PPT, sospecha de PPT, diagnóstico de PPT o en quienes haya sido necesario planear un PPT (así como a sus familiares), que incluya:

- Dar información oral y escrita

- Describir los síntomas y signos de la labor de PPT

- Explicar a la mujer el cuidado que se le podría ofrecer

- Dar información sobre probabilidad de sobrevida y otros riesgos a corto y largo plazo del recién nacido (RN), para lo cual se deberán expresar las cifras como frecuencias naturales (por ejemplo: 1 en 100 en vez de 1\%)

- Informar sobre el cuidado del recién nacido pretérmino (RN PT) incluyendo el establecimiento de salud al cual debe acudir para su cuidado

- Informar sobre los problemas inmediatos que se pueden presentar en un RN PT

- Informar sobre las posibles consecuencias a largo plazo de la prematuridad (cómo crecen y se desarrollan los RN PT)

- Informar sobre cómo la gestante puede establecer su posición sobre la resucitación del RN. Fomentar constantemente posibilidad para hablar y realizar preguntas sobre el tema.

- Cuando sea posible, brindar la oportunidad de visitar la unidad de cuidados neonatales

- Brindar la oportunidad de hablar con un neonatólogo o pediatra.

Considerar que la mujer (y su familia) pueden estar especialmente ansiosos

La identificación del cérvix corto ( $25 \mathrm{~mm}$ ) debería realizarse en la ecografía trasvaginal del segundo trimestre, entre las 18 y 22 semanas de gestación.

Pregunta 2. En gestantes en las que se sospeche de ruptura prematura de membranas (RPM) pretérmino, ¿qué prueba se debería usar para realizar su diagnóstico?

En gestantes con síntomas sugerentes de RPM pretérmino, realizar especuloscopía y, de observarse líquido amniótico, diagnosticar RPM pretérmino sin realizar otras pruebas para confirmar el diagnóstico de RPM.

En las pacientes en quienes no se observa pérdida de líquido amniótico al examen con espéculo, se sugiere complementar con otras pruebas de ayuda diagnóstica, y manejar en base al criterio del médico tratante.

Es importante la evaluación para la inclusión de la disponibilidad de IGFBP-1 o PAMG-1 para su uso en EsSalud. Pregunta 3. En gestantes en labor de PPT, ¿se debería brindar antibióticos profilácticos antes del parto?

En gestantes en labor de PPT con membranas intactas sin signos clínicos de infección, recomendamos no administrar antibióticos de manera rutinaria.

En gestantes con RPM pretérmino recomendamos administrar antibióticos.

En gestantes con RPM pretérmino sugerimos usar eritromicina como antibiótico de elección, a dosis de $250 \mathrm{mg}$ vía oral cuatro veces por día, por diez días.

En gestantes con RPM pretérmino recomendamos no usar la combinación de amoxicilina + ácido clavulánico.

En gestantes con RPM pretérmino, en quienes el médico tratante tenga sospecha bacteriológica que justifique añadir ampicilina, brindar combinación de ampicilina y eritromicina por lo menos durante dos días, seguido por amoxicilina y eritromicina oral.

En gestantes con RPM pretérmino se debería monitorizar la presencia de signos de corioamnionitis y otras infecciones. Para ello, se debe combinar la evaluación clínica con proteína C reactiva, leucocitos y medición de latidos fetales con cardiotocografía. Tener en cuenta que cada prueba por separado no es suficiente para confirmar o excluir la infección intrauterina.

Si los resultados de la evaluación clínica o cualquiera de las pruebas no son consistentes entre ellos, se deberá observar a la paciente y considerar repetir las pruebas.

Recomendación Muy baja
fuerte a favor $(\oplus \Theta \Theta \Theta)$

Recomendación

Moderada favor

$(\oplus \oplus \oplus \Theta)$
$\mathrm{BPC}$

$\mathrm{BPC}$

Pregunta 4. En gestantes con membranas intactas y síntomas de labor de PPT, ¿cuál es la precisión diagnóstica de las pruebas para diagnosticar labor de PPT?

En gestantes con síntomas de labor de PPT (contracciones uterinas) y membranas intactas, recomendamos realizar ecografía trasvaginal para medir la longitud cervical.
BPC

BPC

$\mathrm{NI}$

$\begin{array}{cc}\begin{array}{cc}\text { Recomendación } \\ \text { fuerte en contra }\end{array} & \text { Moderada } \\ \begin{array}{cc}\text { Recomendación } \\ \text { fuerte a favor }\end{array} & (\oplus \oplus \oplus \Theta) \\ \text { Beja } & (\oplus \Theta \Theta) \\ \begin{array}{cc}\text { Recomendación } \\ \text { condicional a } \\ \text { favor }\end{array} & \text { Moderada } \\ \text { Recomendación } & \text { Moderada } \\ \text { fuerte en contra } & (\oplus \oplus \oplus \Theta)\end{array}$

$\mathrm{BPC}$

$\mathrm{BPC}$

$\mathrm{BPC}$ fuerte a favor $(\Theta \Theta \Theta \Theta)$

(Continúa en la pág. 50) 
Tabla 1. Lista de preguntas clínicas, recomendaciones, puntos de buena práctica clínica y notas de implementación (viene de la pág. 49).

\begin{tabular}{|c|c|c|}
\hline Enunciado & Tipo * & $\begin{array}{c}\text { Certeza de la } \\
\text { evidencia }+\end{array}$ \\
\hline Si la longitud cervical es menor a $15 \mathrm{~mm}$, recomendamos seguir el protocolo de PPT. & $\begin{array}{l}\text { Recomendación } \\
\text { fuerte a favor }\end{array}$ & $\begin{array}{l}\text { Muy baja } \\
(\bigoplus \Theta \Theta \Theta)\end{array}$ \\
\hline Si la longitud cervical se encuentra entre 15 a 30mm, recomendamos considerar seguir el protocolo de PPT. & $\begin{array}{l}\text { Recomendación } \\
\text { fuerte a favor }\end{array}$ & $\begin{array}{l}\text { Muy baja } \\
(\bigoplus \Theta \Theta \Theta)\end{array}$ \\
\hline Si la longitud cervical es mayor a 30mm, sugerimos no seguir el protocolo de PPT. & $\begin{array}{l}\text { Recomendación } \\
\text { condicional a favor }\end{array}$ & $\begin{array}{l}\text { Muy baja } \\
(\oplus \Theta \Theta \Theta)\end{array}$ \\
\hline
\end{tabular}

Las ecografías deben ser realizadas por profesional médico entrenado y con experiencia en medición de longitud cervical.

En todas gestantes con sospecha de PPT y edad gestacional menor a 30 semanas, es preferible seguir el protocolo de PPT.

$\mathrm{BPC}$

BPC

\section{Pregunta 5. ¿Cuál es la efectividad clínica del uso de corticoesteroides para maduración fetal pulmonar para mejorar desenlaces maternos y} neonatales?

En mujeres que tienen una edad gestacional confiable de $24^{+0}$ a $33^{+6}$ semanas, en quienes el PPT es considerado inminente y no haya evidencia clínica de infección, recomendamos brindar corticoides prenatales.

Recomendación

fuerte a favor

Recomendación

condicional en contra brindar corticoides prenatales.

Cuando se decida administrar corticoides prenatales, recomendamos brindar un ciclo de dexametasona intramuscular (IM) (6 mg cada 12 horas hasta completar 4 dosis) o de betametasona IM (12 mg cada 24 horas hasta completar 2 dosis).

Si el PPT no ocurre siete días después del primer ciclo completado de corticoides y una evaluación clínica posterior demuestra que hay riesgo de PPT en los siguientes siete días, sugerimos una única repetición del ciclo de corticoides prenatales.

En mujeres elegibles, los corticoides prenatales deben ser administrados cuando el PPT se considere inminente en los próximos siete días del inicio del tratamiento, incluso si se considera inminente en las próximas 24 horas.

Pregunta 6. ¿Cuál es la efectividad clínica de usar sulfato de magnesio (MgSO4) en mujeres en riesgo de PPT para prevenir parálisis cerebral y otros desórdenes neurológicos en niños nacidos pretérmino?

En mujeres con $24^{+0}$ a $29^{+6}$ semanas de gestación en labor de PPT establecido o PPT planificado en las siguientes 24 horas, recomendamos brindar sulfato de magnesio endovenoso.

En mujeres con $30^{+0}$ a $33^{+6}$ semanas de gestación en labor de PPT establecido o PPT planificado en las siguientes 24 horas, sugerimos brindar sulfato de magnesio endovenoso

En los dos casos mencionados, la posología del sulfato de magnesio endovenoso será la siguiente: $4 \mathrm{~g}$ en bolo en 15 minutos, seguido por $1 \mathrm{~g}$ por hora hasta el nacimiento o por 24 horas (lo que suceda primero).

En mujeres que están recibiendo sulfato de magnesio, monitorizar signos clínicos de toxicidad de magnesio por lo menos cada 4 horas, incluyendo reflejos profundos (p. ej.: patelares), estado del sensorio, frecuencia cardíaca, frecuencia respiratoria, presión arterial y diuresis. Si la mujer tiene o desarrolla signos de toxicidad por sulfato de magnesio, monitorizar más frecuentemente la toxicidad y considerar reducir su dosis.

Pregunta 7. ¿Cuál es la efectividad clínica de los tocolíticos en mujeres con labor de PPT sospechado o confirmado para mejorar desenlaces maternos y neonatales?

En mujeres con amenaza de labor de PPT entre las $24^{+0}$ y $33^{+6}$ semanas, sugerimos brindar terapia tocolítica por 48 horas con la finalidad de procurar retrasar el parto, lo cual permitirá administrar terapia con corticoesteroides $\mathrm{y} / 0$ transferir al tercer nive Los bloqueadores de canales de calcio (nifedipino) son los agentes tocolíticos de primera elección.

Recomendación

fuerte a favor

Recomendación condicional a favor

Moderada $(\oplus \oplus \oplus \Theta)$

Muy baja

$(\oplus \Theta \Theta \Theta)$ $(\oplus \oplus \Theta \Theta)$

$\mathrm{BPC}$
Ваја

Recomendación

fuerte a favor

Recomendación condicional a favor

Ваја $(\oplus \oplus \Theta \Theta)$
BPC

$\mathrm{BPC}$

Después de 48 horas de terapia tocolítica, no indique terapia de mantenimiento tocolítica con sulfato de magnesio,

betamiméticos orales, antagonistas de oxitocina ni bloqueadores de canales de calcio. Sin embargo, puede considerar terapia tocolítica de mantenimiento con progesterona vía vaginal.

Pregunta 8. En mujeres con sospecha o diagnóstico de labor de PPT (sin una indicación de cesárea), ¿se debería indicar la realización de cesárea o de parto vaginal?

En mujeres con gestaciones entre las $26^{+0}$ y $36^{+6}$ semanas con presentación en podálico que presenten sospecha o diagnóstico de labor de PPT, considerar la realización de cesárea.

$\mathrm{BPC}$

Pregunta 9. En el RN PT, ¿ise debería realizar un pinzamiento tardío del cordón umbilical?

Si la madre y el RN PT están estables, recomendarnos esperar por lo menos 30 segundos, pero no más de tres minutos, antes de pinzar el cordón.

Si un RN PT debe ser alejado de la madre para resucitación o hay sangrado materno significativo o cualquier otra emergencia que lo amerite, considerar ordeñar y pinzar el cordón lo antes posible.

Posicionar al RN a la altura de o por debajo de la placenta antes de pinzar el cordón.

Como parte del cuidado de RN con peso al nacer $<2000 \mathrm{~g}$, sugerir el uso del método de "mamá canguro" apenas el RN esté clínicamente estable. Si no es posible realizar este método de "manera continuada", también es útil realizarlo de "manera intermitente".

Si el RN con peso al nacer < $2000 \mathrm{~g}$ no puede recibir el método de "mamá canguro", brindarle atención en un ambiente termo-neutral, ya sea con radiadores de calefacción o incubadoras.

Durante la estabilización y transferencia del RN PT de 32 semanas o menos a unidades especializadas, envolverlo con

cobertor plástico para prevenir hipotermia.

$\begin{array}{lc}\text { Recomendación } & \text { Muy baja } \\ \text { fuerte a favor } & (\bigoplus \Theta \Theta \Theta)\end{array}$

BPC

$\mathrm{BPC}$

$\mathrm{BPC}$

$B P C$

$\mathrm{BPC}$

(Continúa en la pág. 51) 
Tabla 1. Lista de preguntas clínicas, recomendaciones, puntos de buena práctica clínica y notas de implementación (viene de la pág. 50).

\section{Enunciado}

Tipo * Certeza de

Pregunta 10. En RN PT con síndrome de distrés respiratorio, ¿se debería usar terapia con presión positiva continua? Para el manejo de RN PT con SDR, recomendamos brindar terapia con presión positiva continua en vía aérea desde sala de partos.

$\begin{array}{cc}\begin{array}{c}\text { Recomendación } \\ \text { fuerte a favor } \\ \text { BPC }\end{array} & \begin{array}{c}\text { Baja } \\ (\oplus \oplus \Theta \Theta)\end{array} \\ \text { BPC } & \\ \text { BPC } & \\ \begin{array}{c}\text { Recomendación } \\ \text { fuerte a favor } \\ \text { BPC }\end{array} & \begin{array}{c}\text { Muy baja } \\ (\bigoplus \Theta \Theta \Theta)\end{array}\end{array}$

La terapia con presión positiva continua en vía aérea debe iniciarse tan pronto como se haga el diagnóstico de SDR en RN PT.

Considerar administrar terapia de reemplazo con surfactante de rescate temprano (derivado de animales o sintéticos con

proteínas) en las primeras dos horas después del parto en RN PT con SDR, cuando sean atendidos en establecimientos de

salud con capacidad de manejo.

No se debe administrar surfactante de manera profiláctica (antes del inicio del SDR en RN PT).

Pregunta 11. En RN PT nacidos antes de las 35 semanas, ¿cuál es la terapia con oxígeno óptima?

Para la estabilización inmediata del RN de 35 semanas de gestación o menos, recomendamos usar terapia con oxígeno entre

$21 \%$ - 30\% (o aire, si no se puede mezclar oxígeno).

Posteriormente, la terapia con oxígeno se guiará según objetivo de saturación usando un oxímetro de pulso.

$\mathrm{BPC}$

* Punto de buena práctica clínica (BPC) o nota de implementación (NI)

† La certeza de la evidencia solo se establece para las recomendaciones y no para los puntos de BPC

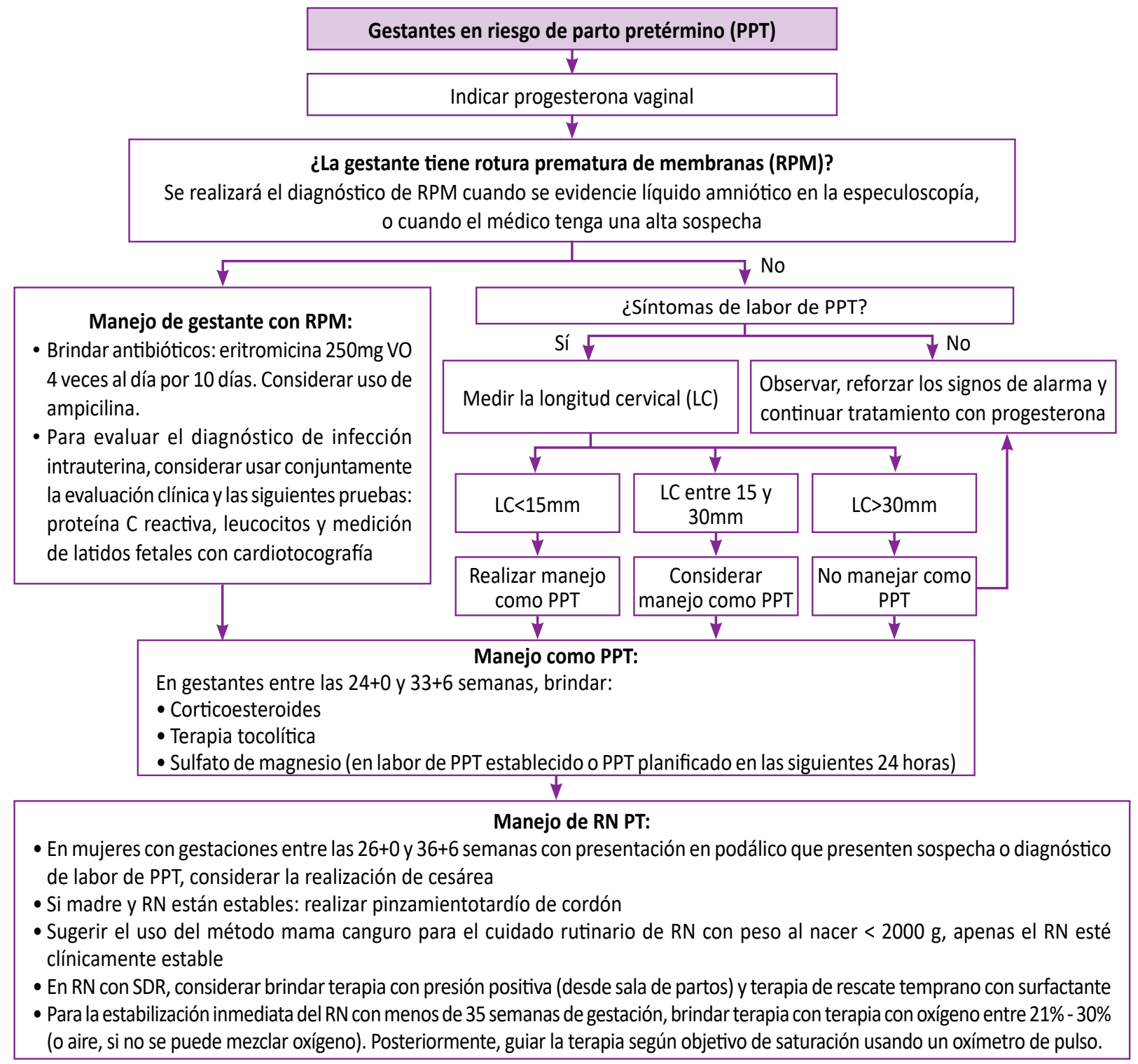

Figura 1. Flujograma de prevención y manejo inicial del parto pretérmino. 
Tabla 2. Significado de los niveles de calidad de la evidencia y de la fuerza de la recomendación.

\section{Significado}

Certeza de la evidencia

$(\oplus \oplus \oplus \oplus)$ Alta

$(\oplus \oplus \oplus \Theta)$ Moderada

$(\oplus \oplus \Theta \Theta)$ Baja

$(\oplus \Theta \Theta \Theta)$ Muy baja

Fuerza de la recomendación

Recomendación fuerte

(a favor o en contra)

Recomendación condicional (a favor o en contra)
Es muy probable que el verdadero efecto sea similar al efecto estimado.

Es moderadamente probable que el verdadero efecto sea similar al efecto estimado, pero es posible que sea sustancialmente diferente

Nuestra confianza en el efecto es limitada. El verdadero efecto podría ser sustancialmente diferente al efecto estimado

Nuestra confianza en el efecto es pequeña. El verdadero efecto probablemente sea sustancialmente diferente al efecto estimado

El GEG-Local cree que todos o casi todos los profesionales que revisan la evidencia disponible seguirían esta recomendación. En la formulación de la recomendación se usa el término "se recomienda"

El GEG-Local cree que la mayoría de los profesionales que revisan la evidencia disponible seguirían esta recomendación, pero un grupo de profesionales podrían no seguirla. En la formulación de la recomendación se usa el término "se sugiere"
Si bien las pruebas tuvieron unas sensibilidades y especificidades altas, la AFP solo fue evaluada en un estudio y ninguna de ellas está disponibles en Perú, por lo que se decidió emitir una NI recomendando que se establezca el uso de las pruebas IGFBP-1 y PAMG-1. Además, se emitieron dos puntos de BPC sobre el diagnóstico clínico de RPM pretérmino y el uso de pruebas complementarias en pacientes con examen físico negativo.

Pregunta 3. En gestantes en labor de PPT, ¿̇se debería brindar antibióticos profilácticos antes del parto?

El GEG consideró dos escenarios: PPT con membranas intactas, y PPT con RPM. Además, comparó regímenes de tratamiento antibiótico.

En mujeres con PPT con membranas intactas, la RS de Flenady $2013^{[12]}$ encontró que el tratamiento antibiótico aumentaba el riesgo de muerte neonatal, disminuía el riesgo de infección materna y no mostraba efectos para otros desenlaces maternos y perinatales adversos. Debido al incremento en el riesgo de muerte neonatal, se decidió emitir una recomendación en contra. Debido a que la certeza de la evidencia en torno a muerte neonatal fue alta, esta recomendación fue fuerte.

En mujeres con PPT y RPM, la RS de Kenyon $2013^{[13]}$ encontró que el tratamiento antibiótico disminuyó el riesgo de corioamnionitis, de neumonía en el RN, de hemocultivo positivo, y de anormalidades cerebrales mayores; y no tuvo efecto sobre la mortalidad materna ni perinatal ni otros desenlaces maternos ni perinatales adversos. Es decir, en este grupo los beneficios fueron superiores a los riesgos, por lo que se decidió emitir una recomendación a favor del tratamiento antibiótico. Debido a que la certeza de la evidencia es moderada a alta, la recomendación fue fuerte.
En relación a los regímenes de tratamiento antibiótico, la RS realizada por la GPC OMS $2015^{\left[{ }^{6]}\right.}$ sugiere que la eritromicina tendría mayores beneficios y menos riesgos que otros regímenes evaluados, por lo que se emite una recomendación a favor de usar este antibiótico. A pesar que la certeza de la evidencia es moderada, la elección del régimen antibiótico podría variar según las características de la paciente y el perfil de resistencia antibiótica del establecimiento, por lo cual esta recomendación es condicional.

Por otro lado, la misma RS encontró que la amoxicilina + ácido clavulánico tenía más efectos adversos (enterocolitis necrotizante) que otros regímenes, por lo que se emite una recomendación en contra de su uso. Al tener una certeza moderada, esta recomendación fue fuerte.

Asimismo, se consideró emitir tres puntos de BPC sobre los criterios para indicar los regímenes antibióticos (de incluir ampicilina en el régimen de ser necesario y justificado, con base en lo indicado por la GPC de la American College of Obstetricians and Gynecologists ${ }^{[14]}$ ), el procedimiento para diagnosticar corioamnionitis con base a la GPC NICE $2015^{[5]}$ y la postura ante una evaluación clínica y/o pruebas diagnósticas inconsistentes.

Pregunta 4. En gestantes con membranas intactas y síntomas de labor de PPT, ¿¿cuál es la precisión diagnóstica de las pruebas para diagnosticar labor de PPT?

El GEG decidió evaluar la longitud cervical (LC) y la fibronectina (marcador bioquímico) como criterios diagnósticos para predecir la progresión del trabajo de parto en gestantes con sintomatología de trabajo de PPT (contracciones uterinas con membranas intactas). 
En la predicción de PPT, a las 48 horas de inicio de sintomatología, la RS de Boots $2014^{[15]}$ encontró que la fibronectina tuvo una sensibilidad de $62 \%$ y una especificidad de $81 \%$, mientras que la $\mathrm{LC}<15 \mathrm{~mm}$ tuvo una sensibilidad de $77 \%$ y especificidad de $88 \%$. Asimismo, para la predicción de PPT, a los siete días de inicio de sintomatología, la fibronectina tuvo una sensibilidad de $75 \%$ y una especificidad de $79 \%$, la LC $<15 \mathrm{~mm}$ tuvo una sensibilidad de $74 \%$ y especificidad de $89 \%$ y la $\mathrm{LC}<30 \mathrm{~mm}$ tuvo una sensibilidad de $95 \%$ y una especificidad de $46 \%$.

Ya que la fibronectina no es superior, en términos de sensibilidad o especificidad, que la LC y que no está disponible se decidió emitir una recomendación a favor del uso de la LC como criterio diagnóstico. A pesar que la evidencia fue de muy baja certeza, se consideró que inclinarse por otras pruebas no era coherente a la luz de la evidencia actual, por lo cual esta recomendación fue fuerte.

La sensibilidad y especificidad de la LC $<15 \mathrm{~mm}$ fue aceptable para predecir PPT a los siete días de inicio de la sintomatología; por eso, se decidió emitir una recomendación a favor de seguir el protocolo de PPT y, a pesar que la certeza de la evidencia era muy baja, considerarla como fuerte por el daño que se ocasionaría al no actuar ante esta situación.

A pesar de que la especificidad de la $\mathrm{LC}<15 \mathrm{~mm}$ fue buena, su sensibilidad no, por lo que las gestantes con un valor de LC mayor podrían estar en riesgo de progresar a PPT sin ser oportunamente diagnosticadas. Por ello, se emitió una recomendación a favor de considerar seguir el protocolo existente de PPT en casos de LC entre 15 y $30 \mathrm{~mm}$. Para evitar daños al no dar tratamiento a estas pacientes esta recomendación fue fuerte.

La especificidad de la $\mathrm{LC}<30 \mathrm{~mm}$ fue baja, por lo que se emitió una recomendación en contra, pero dada la muy baja certeza de la evidencia, esta recomendación fue condicional.

Además, el GEG emitió dos puntos de BPC sobre la necesaria capacitación del personal de salud encargado del diagnóstico de LC por ecografía trasvaginal y la adopción de la GPC NICE en cuanto al manejo de pacientes con menos de 30 semanas de gestación.

Pregunta 5. ¿Cuál es la efectividad clínica del uso de corticoesteroides para maduración fetal pulmonar para mejorar desenlaces maternos y neonatales?

El GEG evaluó la eficacia del uso de corticoesteroides para la maduración pulmonar en dos grupos de gestantes (con sospecha de PPT de $24^{+0}$ a $33^{+6}$ semanas y con sospecha de PPT de $34^{+0}$ a $36^{+6}$ semanas que serán sometidas a cesárea) así como los regímenes de tratamiento.

En gestantes con trabajo de PPT la RS de Roberts $201{ }^{\text {[16] }}$ encontró que la administración de corticoesterorides disminuyó el riesgo de muerte perinatal y neonatal, y otros desenlaces perinatales adversos, aunque no tuvo efecto sobre desenlaces maternos. Así, se consideró que la terapia tuvo grandes beneficios y en la bibliografía consultada no se encontraron daños, por lo que se emitió una recomendación a favor del uso de corticoesteroides en este grupo. Debido a que la certeza de evidencia fue moderada, la recomendación fue fuerte.

En gestantes sometidas a cesárea electiva entre las 34 y $36^{+6}$ semanas, la RS de Sotiriadis $2009{ }^{[17]}$ encontró un solo ensayo clínico aleatorizado (ECA) que reportó que los corticoesteroides disminuyeron el riesgo de admisión a la unidad de cuidados intensivos ( $\mathrm{UCl}$ ) por morbilidad respiratoria, aunque no tuvieron efecto en otros desenlaces, tampoco se presentaron otros efectos adversos. Por ello, el GEG consideró que aún no se cuenta con un balance de beneficios y riesgos suficiente para aplicar esta intervención de manera universal, y decidió emitir una recomendación en contra. Sin embargo, al considerar que en algunos casos el médico tratante puede considerar beneficiosa esta terapia, la recomendación fue condicional.

En relación a los regímenes de corticoesteroides, la RS de Brownfoot $2013^{[18]}$ no encontró diferencias entre la dexametasona y la betametasona en cuanto a riesgo de muerte neonatal ni otros desenlaces perinatales adversos. Sin embargo, encontró un mayor riesgo de sepsis neonatal al usar dexametasona oral en comparación con dexametasona intramuscular. De esta manera, se decidió emitir una recomendación a favor del uso intramuscular de dexametosona o betametasona. Puesto que la certeza de evidencia fue moderada y los potenciales beneficios eran muy altos, esta recomendación fue fuerte.

En relación a la comparación entre curso único y cursos repetidos de corticoesterorides, la RS de Crowther $2011^{[19]}$ encontró que los cursos únicos tuvieron un menor riesgo de desarrollar SDR, aunque no se encontraron diferencias en el riesgo de mortalidad neonatal o fetal ni otros desenlaces perinatales adversos. Por ello, se emitió una recomendación a favor del uso de cursos únicos. Debido la baja certeza de la evidencia, esta recomendación fue condicional.

Además el GEG emitió un punto de BPC sobre el momento en que deben ser administrados los corticoesterorides ${ }^{[6]}$.

Pregunta 6. ¿Cuál es la efectividad clínica de usar sulfato de magnesio (MgSO4) en mujeres con riesgo de PPT para prevenir parálisis cerebral y otros desórdenes neurológicos en niños nacidos pretérmino?

Se evaluó la efectividad del $\mathrm{MgSO} 4$ en la neuroprotección de RN PT en dos grupos: de $24^{+0}$ a $29^{+6}$ semanas y de $30^{+0}$ a $33^{+6}$ semanas. Para ello, se usó la RS de la GPC NICE $2015^{[5]}$.

En mujeres en riesgo de PPT (hasta antes de las 34 semanas) se encontró una reducción en el riesgo de desarrollar parálisis cerebral en el RN y de diagnóstico de parálisis cerebral a los dos años de edad, pero no tuvo efecto en cuanto a riesgo de óbito fetal ni otros desenlaces perinatales adversos. No obstante, esta 
intervención aumentó el riesgo de la aparición de cualquier efecto adverso que provoque la interrupción de la infusión o el descenso de la presión arterial diastólica en la madre, mas no se encontró efecto en hipotensión.

Por otro lado, al hacer un análisis adicional en el subgrupo de mujeres en riesgo de PPT después de las 30 semanas de gestación - que sólo incluye a un ECA-, el MgSO4 no tuvo efecto sobre el riesgo de parálisis cerebral en hijos PT. Debido a la evidencia contradictoria, se decidió emitir dos recomendaciones teniendo en cuenta el punto de corte de 30 semanas de gestación.

En el subgrupo de mujeres en riesgo de PPT de $24^{+0}$ a $29^{+6}$ semanas se encontró que los beneficios superaban los riesgos en la madre, por lo cual, se emitió una recomendación a favor del uso de MgSO4. A pesar de la baja certeza de la evidencia, el GEG consideró que los beneficios potenciales eran muy importantes, por lo que esta recomendación fue fuerte.

En el subgrupo de mujeres en riesgo de PPT de $30^{+0}$ a $33^{+6}$ semanas se encontró un solo ECA, el cual reportó que la intervención con MgSO4 no tuvo efecto en la incidencia de parálisis cerebral en RN PT. Debido a que la certeza fue baja y a que solo se encontró un ECA, el GEG consideró considerar los beneficios encontrados en gestantes de $24^{+0}$ a $29^{+6}$ semanas, por lo que emite una recomendación a favor del uso de MgSO4. Puesto a que la evidencia fue de baja certeza y que proviene de un solo ECA, fue una recomendación condicional.

Además, el GEG emitió dos puntos de BPC en relación a la posología del $\mathrm{MgSO} 4$ y a la monitorización de los signos de toxicidad.

Pregunta 7. ¿Cuál es la efectividad clínica de los tocolíticos en mujeres con labor de PPT sospechado o confirmado para mejorar desenlaces maternos y neonatales?

El GEG evaluó la efectividad del uso los tocolíticos en el retraso de parto en gestantes con amenaza o trabajo de PPT, lo que permitiría otras intervenciones y prevenir desenlaces maternos y perinatales adversos. Para ello, se consideró la RS de GPC KCE $2014^{[7]}$.

En gestantes con amenaza o trabajo de PPT se encontró que los betamiméticos, inhibidores de prostaglandinas, bloqueadores de canales de calcio, sulfato de magnesio y bloqueadores de receptores de oxitocina disminuyeron el riesgo de ocurrencia de parto dentro de las 48 horas -retrasaron el parto-, aunque, en el RN PT, no tuvieron efecto sobre el riesgo de SDR, mortalidad neonatal ni otro evento adverso ${ }^{[7]}$. Por ello, se emitió una recomendación a favor de brindar tocolíticos. Sin embargo, debido a que la evidencia se basa en comparaciones indirectas, esta recomendación fue condicional.

En cuanto al tocolítico de primera elección se estableció un punto de BPC sobre brindar bloqueadores de canales de calcio (nifedipino) como tocolítico por tener mínimos efectos adversos y ser de fácil administración; además, se emitió otro punto de BPC sobre la terapia tocolítica de mantenimiento.

Pregunta 8. En mujeres con sospecha o diagnóstico de labor de PPT (sin una indicación de cesárea), ¿se debería indicar la realización de cesárea o de parto vaginal?

En mujeres con embarazo único, con sospecha o en trabajo de PPT, la RS de Alfirevic $2013^{[20]}$ encontró que la realización de cesárea aumentó el riesgo de infección materna pero no tuvo efecto sobre muerte perinatal, morbilidad neonatal severa, infección de herida ni estancia hospitalaria mayor a diez días, en comparación con mujeres que tuvieron parto vaginal. Si bien se espera un mayor riesgo de infección materna cuando se realiza cesáreas, el GEG consideró que aún no había evidencia clara de los posibles beneficios y concluyó que la evidencia actual no permitía emitir una recomendación al respecto.

Sin embargo, el GEG consideró importante realizar la pregunta en el grupo de mujeres con fetos en posición podálica. Se decidió emitir un punto de BPC a favor de la cesárea con base en la RS de Bergenhenegouwen $2014{ }^{[21]}$ que encontró siete estudios observacionales y reportó que la cesárea disminuyó el riesgo de mortalidad neonatal y total (desenlace compuesto por mortalidad intraparto y neonatal).

Pregunta 9. En el RN PT, ¿se debería realizar una pinzamiento tardío del cordón umbilical (CU)?

Al evaluar la eficacia del pinzamiento (en inglés: clamping) tardío de CU (realizado después de 30 segundos y hasta 3 minutos después del parto) en RN PT, la RS de la GPC NICE $2015^{[5]}$ encontró que esta intervención redujo el riesgo de hemorragia intraventricular y de requerimiento de transfusión por anemia en neonatos, aumentó el valor de hematocrito a las cuatro y 24 horas desde el nacimiento y no tuvo efecto en otro desenlaces perinatales adversos, en comparación con la realización de pinzamiento temprano (desde 5 hasta 30 segundos después del nacimiento). Por ende, se emitió una recomendación $a$ favor del pinzamiento tardío y, a pesar que la evidencia tenía baja certeza, se consideró que los beneficios potenciales eran grandes y los riesgos potenciales despreciables, por lo que esta recomendación fue fuerte.

Asimismo, se emitió cinco puntos de BPC en relación el pinzamiento en casos de emergencia, la posición del RN antes del pinzamiento, el cuidado térmico en RN PT y el método "mamá canguro" ${ }^{[6]}$.

Pregunta 10. En RN PT con síndrome de distrés respiratorio, ¿'se debería usar terapia con presión positiva continua (CPAP)?

El GEG evaluó la eficacia de la terapia con presión positiva continua en vía aérea (CPAP) en RN PT con SDR. Para ello, la RS de Ho $2002^{[22]}$ encontró que la administración de CPAP disminuyó el riesgo de muerte neonatal y de falla respiratoria que necesitase ventilación asistida, no tuvo efecto sobre el riesgo de necesitar 
surfactante o de desarrollar displasia bronco pulmonar (DBP) pero aumentó el riesgo de neumotórax, todos en comparación con los RN PT que recibieron solo oxígeno. Por ello, se decidió emitir una recomendación a favor del uso de CPAP. A pesar que la certeza de la evidencia fue baja, se consideró que los beneficios fueron considerados importantes, por lo que esta recomendación fue fuerte.

Además, el GEG emitió tres puntos de BPC con respecto a inicio de la terapia CPAP y el uso de terapia de reemplazo con surfactante ${ }^{[6]}$.

Pregunta 11. En RN PT nacidos antes de las 35 semanas, ¿'cuál es la terapia con oxígeno óptima?

El GEG comparó la eficacia de la administración de oxigenoterapia a bajas y altas concentraciones para la estabilización inmediata de los RN PT nacidos antes de las 35 semanas. Para ello, la RS de Brown $2012^{[23]}$ encontró que la administración de oxigenoterapia a bajas dosis disminuyó el riesgo de muerte neonatal y no tuvo efecto sobre el riesgo de DBP ni otros desenlaces perinatales adversos, en comparación con los RN PT que recibieron concentraciones altas de oxígeno. La proporción de RN que alcanzaron el objetivo en la saturación de oxígeno 10 minutos después del nacimiento y la duración de ventilación mecánica o necesidad de intubación endotraqueal durante la resucitación fueron similares en ambos grupos. A pesar de no encontrar diferencias en varios desenlaces perinatales, el GEG consideró que la reducción del riesgo de mortalidad neonatal es muy importante, por lo que se emitió una recomendación a favor del uso de oxigenoterapia a bajas concentraciones (21\% - 30\% según los grandes estudios de la RS). A pesar que la certeza de la evidencia fue muy baja, la terapia es de fácil aplicación, poco costosa y potencialmente beneficiosa por lo que esta recomendación fue fuerte.

Además, el GEG emitió un punto de BPC en relación al seguimiento de la oxigenoterapia.

Agradecimientos: Al Dr. Enrique Gil Guevara (director médico del Instituto Peruano de Medicina y Cirugía Fetal, ORCID iD: 0000-0001-6515-2599), al Dr. Max Robert Roetter Palomino (médico pediatra neonatólogo del Hospital Nacional Dos de Mayo de Lima, Perú) y al Dr. José Pacheco Romero (coordinador del Grupo de Investigación en Medicina y Genética Molecular Materno Perinatal de la Universidad Nacional Mayor de San Marcos, ORCID iD: 0000-0002-3168-6717) por la revisión que realizaron a la presente guía.

Contribuciones de autoría: Todos los autores participaron en la realización de la guía. LO, AGG, JHZT y ATR realizaron la búsqueda de información bibliográfica y evaluaron la calidad de los estudios para cada pregunta. NBC redactó la primera versión del artículo. Todos los autores participaron en la discusión de los estudios encontrados y la formulación de las recomendaciones y puntos de buenas prácticas clínicas. Todos los autores revisaron y aprobaron la versión final del artículo.
Financiamiento: Este documento técnico ha sido financiado por el IETSI de EsSalud.

\section{Conflictos de interés: Ninguno.}

\section{REFERENCIAS BIBLIOGRÁFICAS}

1. Blencowe $H$, Cousens $S$, Oestergaard MZ, Chou D, Moller AB, Narwal R, et al. National, regional, and worldwide estimates of preterm birth rates in the year 2010 with time trends since 1990 for selected countries: a systematic analysis and implications. Lancet. 2012;379(9832):2162-72.

2. Ministerio de Salud del Perú. Boletín Epidemiológico del Perú Vol 26 - SE 492017 [Internet]. Lima, Perú: Ministerio de Salud; 2017 [citado 7 Dic 2018]. Disponible en: http://www.dge.gob. pe/portal/docs/vigilancia/boletines/2017/49.pdf.

3. Beck S, Wojdyla D, Say L, Betran AP, Merialdi M, Requejo JH, et al. The worldwide incidence of preterm birth: a systematic review of maternal mortality and morbidity. Bull World Health Organ. 2010;88(1):31-8.

4. lams JD, Romero R, Culhane JF, Goldenberg RL. Primary, secondary, and tertiary interventions to reduce the morbidity and mortality of preterm birth. Lancet. 2008;371(9607):164-75.

5. National Institute for Clinical Excellence. Clinical Guideline 25 Preterm Labour and Birth. London: NICE; 2015.

6. World Health Organization. WHO recommendations on interventions to improve preterm birth outcomes: evidence base. $1^{\circ}$ ed. Geneva: World Health Organization; 2015

7. Roelens K, Roberfroid D, Ahmadzai N, Ansari M, Singh K, Gaudet $L$, et al. Prevention of preterm birth in women at risk: selected topics - Abstract. Good Clinical Practice (GCP). Brussels: Belgian Health Care Knowledge Centre (KCE). 2014. KCE Reports 228Cs. D/2014/10.273/62.

8. Andrews J, Guyatt G, Oxman AD, Alderson P, Dahm P, FalckYtter $Y$, et al. GRADE guidelines: 14. Going from evidence to recommendations: the significance and presentation of recommendations. J Clin Epidemiol. 2013;66(7):719-25.

9. Romero R, Nicolaides K, Conde-Agudelo A, Tabor A, O'brien $J M$, Cetingoz $E$, et al. Vaginal progesterone in women with an asymptomatic sonographic short cervix in the midtrimester decreases preterm delivery and neonatal morbidity: a systematic review and metaanalysis of individual patient data. Am J Obstet Gynecol. 2012;206(2):124.e1-19.

10. Palacio $M$, Kuhnert $M$, Berger $R$, Larios $C L$, Marcellin $L$. Meta-analysis of studies on biochemical marker tests for the diagnosis of premature rupture of membranes: comparison of performance indexes. BMC Pregnancy Childbirth. 2014;14:183.

11. Eleje GU, Ezugwu EC, Eke AC, Ikechebelu JI, Ezeama CO, Ezebialu $I U$, et al. Accuracy and response time of dual biomarker model of insulin-like growth factor binding protein-1/ alpha fetoprotein (Amnioquick duo+) in comparison to placental alpha-microglobulin-1 test in diagnosis of premature rupture of membranes. J Obstet Gynaecol Res. 2017;43(5):825-33.

12. Flenady V, Hawley G, Stock OM, Kenyon S, Badawi N. Prophylactic antibiotics for inhibiting preterm labour with intact membranes. Cochrane Database Syst Rev. 2013;(12):CD000246.

13. Kenyon SL, Taylor DJ, Tarnow-Mordi W; ORACLE Collaborative Group. Broad-spectrum antibiotics for spontaneous preterm labour: the ORACLE II randomised trial. ORACLE Collaborative Group. Lancet. 2001;357(9261):989-94. 
14. American College of Obstetricians and Gynecologists' Committee on Practice Bulletins-Obstetrics. Practice Bulletin clinical management guidelines for obstetrician-gynecologists No. 172: Premature Rupture of Membranes. Obstet Gynecol. 2016;128(4):e165-77.

15. Boots AB, Sanchez-Ramos L, Bowers DM, Kaunitz AM, Zamora J, Schlattmann P. The short-term prediction of preterm birth: a systematic review and diagnostic metaanalysis. Am J Obstet Gynecol. 2014;210(1):54.e1-54.e10.

16. Roberts D, Brown J, Medley N, Dalziel SR. Antenatal corticosteroids for accelerating fetal lung maturation for women at risk of preterm birth. Cochrane Database Syst Rev. 2017;3:CD004454.

17. Sotiriadis A, Makrydimas G, Papatheodorou S, Ioannidis JP. Corticosteroids for preventing neonatal respiratory morbidity after elective caesarean section at term. Cochrane Database Syst Rev. 2009;(4):CD006614.

18. Brownfoot FC, Gagliardi DI, Bain E, Middleton P, Crowther CA. Different corticosteroids and regimens for accelerating fetal lung maturation for women at risk of preterm birth. Cochrane Database Syst Rev. 2013;(8):CD006764.
19. Crowther CA, McKinlay CJ, Middleton P, Harding JE. Repeat doses of prenatal corticosteroids for women at risk of preterm birth for improving neonatal health outcomes. Cochrane Database Syst Rev. 2015;(7):CD003935.

20. Alfirevic Z, Milan SJ, Livio S. Caesarean section versus vaginal delivery for preterm birth in singletons. Cochrane Database Syst Rev. 2012;(6):CD000078

21. Bergenhenegouwen L, Meertens L, Schaaf J, Nijhuis J, Mol B, Kok $M$, et al. Vaginal delivery versus caesarean section in preterm breech delivery: a systematic review. Eur J Obstet Gynecol Reprod Biol. 2014;172:1-6.

22. Ho JJ, Subramaniam P, Henderson-Smart DJ, Davis PG. Continuous distending pressure for respiratory distress syndrome in preterm infants. Cochrane Database Syst Rev. 2002;(2):CD002271.

23. Brown JV, Moe-Byrne T, Harden M, McGuire W. Lower versus higher oxygen concentration for delivery room stabilisation of preterm neonates: systematic review. PLoS One. 2012;7(12):e52033.

\title{
Las ediciones anteriores de Acta Médica Peruana están disponibles en:
}

\author{
www.scielo.org.pe
}

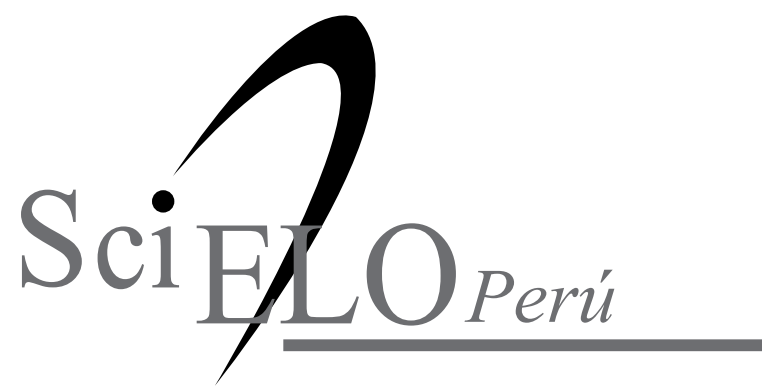

